TRANSACTIONS OF THE

AMERICAN MATHEMATICAL SOCIETY

Volume 359, Number 9, September 2007, Pages 4405-4427

S 0002-9947(07)04235-3

Article electronically published on March 20, 2007

\title{
REPRESENTABLE IDEMPOTENT COMMUTATIVE RESIDUATED LATTICES
}

\author{
J. G. RAFTERY
}

\begin{abstract}
It is proved that the variety of representable idempotent commutative residuated lattices is locally finite. The $n$-generated subdirectly irreducible algebras in this variety are shown to have at most $3 n+1$ elements each. A constructive characterization of the subdirectly irreducible algebras is provided, with some applications. The main result implies that every finitely based extension of positive relevance logic containing the mingle and Gödel-Dummett axioms has a solvable deducibility problem.
\end{abstract}

\section{INTRODUCTION}

A lattice-ordered commutative monoid is an algebra $\langle A ; \cdot, \wedge, \vee, e\rangle$ made up of a commutative monoid $\langle A ; \cdot, e\rangle$ and a lattice $\langle A ; \wedge, \vee\rangle$, where the lattice order $\leq$ is preserved by the functions $x \mapsto a \cdot x$, for every $a \in A$. A structure of this kind is said to be residuated provided that for each pair $a, b \in A$, there is a largest $c \in A$ such that $a \cdot c \leq b$. The largest $c$ with this property is then denoted by $a \rightarrow b$ and the binary operation $\rightarrow$ is called residuation. In this case the algebra $\langle A ; \cdot, \rightarrow, \wedge, \vee, e\rangle$ is called a commutative residuated lattice, or briefly a $C R L$. It follows that every CRL satisfies

$$
z \leq x \rightarrow y \Longleftrightarrow x \cdot z \leq y
$$

A CRL need not be integral; i.e., its monoid identity $e$ need not be its greatest element. Lattice-ordered abelian groups are examples of nonintegral CRLs in which $x \rightarrow y=x^{-1} \cdot y$. They are representable in the sense that they may be embedded into direct products of totally ordered algebras of the same kind.

In a CRL, if $x \leq e$, then clearly $x \cdot x \leq x$. Therefore, an integral CRL satisfies the square increasing law $x \leq x \cdot x$ only if it is idempotent, i.e., satisfies $x \approx x \cdot x$. In an idempotent integral $\mathrm{CRL}, \cdot$ and $\wedge$ coincide, so $\rightarrow$ is just relative pseudocomplementation. The representable CRLs with these properties are the relative Stone algebras of $[5$.

In relevance logic and in classical linear logic, the connective of co-tenability $\neg(p \rightarrow \neg q)$ is distinct from conjunction $p \wedge q$. In Anderson and Belnap's system $\mathbf{R}$, as defined in [3], co-tenability is modeled after the monoid operations of distributive square increasing CRLs with an involution, i.e., de Morgan monoids. Here, residuation models the connective of relevant implication. The idempotent

Received by the editors September 25, 2005.

2000 Mathematics Subject Classification. Primary 03B47, 03G25, 06D99, 06F05, 08A50, $08 \mathrm{C} 15$.

Key words and phrases. Locally finite variety, residuation, residuated lattice, representable, idempotent, Sugihara monoid, relative Stone algebra, relevance logic, mingle.

(C) 2007 American Mathematical Society

Reverts to public domain 28 years from publication 
de Morgan monoids are called Sugihara monoids. These algebras are representable, and not generally integral. They model the system $\mathbf{R} \mathbf{M}^{t}$, which is the extension of $\mathbf{R}$ by the mingle axiom $\vdash p \rightarrow(p \rightarrow p)$.

Of the properties discussed above, the following three will be central to this paper: a CRL is said to be

- linear if its lattice reduct is totally ordered;

- representable if it embeds in a direct product of linear CRLs;

- idempotent if it satisfies $x \cdot x \approx x$.

The class of all representable idempotent CRLs - briefly RICRLs - is a finitely axiomatized variety.

The main result of this paper establishes that the variety of all RICRLs is locally finite (Theorem 18) and, in particular, that an $n$-generated subdirectly irreducible RICRL has at most $3 n+1$ elements (Theorem 17). This explains simultaneously the phenomenon of local finiteness in relative Stone algebras and the same phenomenon in Sugihara monoids. A constructive characterization of the subdirectly irreducible RICRLs will be provided (Theorem 20). It implies that any such algebra is, roughly speaking, an amalgam of a Sugihara monoid and a family of relative Stone algebras (Theorem 21). The result allows us to establish a finite equational basis for the positive Sugihara monoids, i.e., the subalgebras of the involution-free reducts of Sugihara monoids. It also allows us to prove that RICRLs and Sugihara monoids satisfy the same universal sentences in the language of lattice-ordered monoids.

The negation-less fragment of the relevant logic $\mathbf{R}$ is denoted as $\mathbf{R}_{+}$. Theorem 18 implies that the quasi-equational theory of any finitely axiomatized quasivariety of RICRLs is decidable. These quasi-equational theories coincide, up to invertible translation, with the finitely based extensions of $\mathbf{R}_{+}$that contain both the mingle axiom and the Gödel-Dummett axiom $\vdash(p \rightarrow q) \vee(q \rightarrow p)$. Consequently, any such extension of $\mathbf{R}_{+}$is decidable; in fact, it has a solvable deducibility problem. There are infinitely many axiomatic extensions of this kind that are weaker than the negation-less fragment of $\mathbf{R} \mathbf{M}^{t}$ as well as that of the Gödel-Dummett logic $\mathbf{L C}$.

For background in universal algebras, see 14]. For general information about CRLs, see [24, 43, for relevance logic [2, 3], for linear logic [4, 41, and for substructural logics in general, [22, 36, 37, 39]. Further references can be found in the bibliographies of $12,13,25]$.

\section{Algebraic preliminaries}

The universe of an algebra $\boldsymbol{A}$ will usually be denoted as $A$. We shall make standard use, as in [14, of the class operator symbols I, H, S, P, $\mathrm{P}_{\mathrm{S}}$ and $\mathrm{P}_{\mathrm{U}}$. They stand, respectively, for closure under isomorphic and homomorphic images, subalgebras, direct and subdirect products, and ultraproducts. Given a class $\mathrm{X}$ of similar algebras, we use $Q(X)$ and $V(X)$ to denote, respectively, the quasivariety $\operatorname{ISPP}_{U}(X)$ and the variety $\operatorname{HSP}(X)$ generated by $X$. The class of all subdirectly irreducible algebras in $\mathrm{X}$ will be denoted by $\mathrm{X}_{\mathrm{SI}}$.

An algebra is said to be $n$-generated if it has a generating set with at most $n$ elements. A variety or quasivariety of algebras is said to be locally finite if each of its finitely generated members is a finite algebra. A variety is finitely generated if it has the form $\mathrm{V}(\mathrm{X})$ for some finite set $\mathrm{X}$ consisting of finite algebras. Every finitely generated variety is locally finite, but not conversely. A finitely 
axiomatized and locally finite quasivariety $\mathrm{K}$ of finite type must have a decidable quasi-equational theory, because both its valid quasi-identities and its finite models may be enumerated mechanically. In this case, moreover, every finitely axiomatized subquasivariety of $\mathrm{K}$ has a decidable quasi-equational theory, because local finiteness is obviously a hereditary property. In this respect local finiteness is strictly stronger than the finite embeddability property (FEP), which has been exploited for similar purposes in 12, 13, 42. The FEP asks only that every finite subset of an algebra in $\mathrm{K}$ can be extended to a finite algebra in $\mathrm{K}$ in such a way that partial operations are preserved. It is not a hereditary property.

The following well-known result is a standard tool for proving local finiteness in varieties that are not finitely generated. We shall need to rely on it, so we recall the proof for the sake of completeness.

Theorem 1. Let $\mathrm{V}$ be a variety of finite type. Suppose there exists a fixed integervalued function $f$ such that, for each nonnegative integer $n$, it is the case that every $n$-generated subdirectly irreducible algebra in $\mathrm{V}$ has at most $f(n)$ elements. Then $\mathrm{V}$ is locally finite.

Proof. For each positive integer $m$, there are only finitely many distinct isomorphism classes of $m$-element subdirectly irreducible algebras in $\mathrm{V}$, because the type of $\mathrm{V}$ is finite. Let $S(m)$ be a transversal of these isomorphism classes, i.e., $S(m)$ consists of just one $m$-element subdirectly irreducible algebra from each isomorphism class. So $S(m)$ is a finite set. Now let $\boldsymbol{A} \in \mathrm{V}$ be an algebra that is generated by an $n$-element subset $X$ of $A$. Every homomorphic image of $\boldsymbol{A}$ is $n$-generated, so, by assumption, every subdirectly irreducible homomorphic image of $\boldsymbol{A}$ has at most $f(n)$ elements. Thus $\boldsymbol{A}$ may be represented as a subdirect product of algebras in $U(n):=\bigcup_{m \leq f(n)} S(m)$, by Birkhoff's subdirect decomposition theorem. But every homomorphism with domain $\boldsymbol{A}$ is completely determined by its restriction to $X$, and there are only finitely many distinct functions from $X$ into algebras in the finite set $U(n)$. Therefore, we may represent $\boldsymbol{A}$ as an irredundant subdirect product of algebras $\boldsymbol{A}_{i} \in U(n), i \in I$, for some finite index set $I$. Since $\boldsymbol{A}$ embeds in $\prod_{i \in I} \boldsymbol{A}_{i}$, we have $|A| \leq\left|\prod_{i \in I} A_{i}\right| \leq \prod_{i \in I} f(n)=f(n)^{|I|}$, so $\boldsymbol{A}$ is a finite algebra.

Of course the converse of this result is true as well, since every $n$-generated algebra in a variety $\mathrm{V}$ is a homomorphic image of the free $n$-generated algebra in $\mathrm{V}$, which is unique up to isomorphism.

Suppose $\boldsymbol{A}$ is an algebra and $O$ a subset of the signature of $\boldsymbol{A}$. The $O-$ reduct of $\boldsymbol{A}$ is the algebra with the same universe as $\boldsymbol{A}$ whose basic operations are just the interpretations in $\boldsymbol{A}$ of the operation symbols in $O$. The subalgebras of this $O$-reduct are then called the $O$-subreducts of $\boldsymbol{A}$. The class of all $O$-subreducts of algebras in a fixed quasivariety $Q$ is itself a quasivariety (see [28, p. 216]). Clearly, this quasivariety is locally finite if $Q$ is.

An algebra is called a lattice expansion if it has a reduct that is a lattice. Recall that lattices are congruence distributive and that congruence distributivity is a Mal'cev condition. It follows that every variety consisting of lattice expansions is congruence distributive. We shall need to use 'Jónsson's Lemma' and one of its consequences. We recall the following statements. 
Theorem 2 (Jónsson [26], or see [14, Thm. IV.6.10]).

(i) If $\mathbf{X}$ is any subclass of a congruence distributive variety, then

$$
(\mathrm{V}(\mathrm{X}))_{\mathrm{SI}} \subseteq \mathrm{HSP}_{\mathrm{U}}(\mathrm{X}) \text {, whence } \mathrm{V}(\mathrm{X})=\operatorname{IP}_{\mathrm{S}} \mathrm{HSP}_{\mathrm{U}}(\mathrm{X}) \text {. }
$$

(ii) For any two subvarieties $\mathrm{M}$ and $\mathrm{N}$ of a congruence distributive variety,

$$
(\mathrm{V}(\mathrm{M} \cup \mathrm{N}))_{\mathrm{SI}}=\mathrm{M}_{\mathrm{SI}} \cup \mathrm{N}_{\mathrm{SI}} \text {. }
$$

\section{BASIC PROPERTIES OF CRLS}

The class of all CRLs was defined in the introduction, and it is a variety. When only one CRL $\boldsymbol{A}$ is under discussion, its operations and identity will always be denoted by $\cdot, \rightarrow, \wedge, \vee$ and $e$. If $a \in A$, we define

$$
a^{0}:=e \text { and } a^{n+1}:=a^{n} \cdot a \text { for all nonnegative integers } n .
$$

In any CRL, the term function $x \rightarrow y$ is order preserving in its second argument and order reversing in its first, as a consequence of the definition of residuation. For typographical reasons, we shall frequently employ the following abbreviation in the context of CRLs:

Definition 1. $|x|:=x \rightarrow x$.

In the sequel, formal inequations $\alpha \leq \beta$ will often be referred to as 'identities', because they can be taken to abbreviate equations $\alpha \approx \alpha \wedge \beta$. We list some well-known identities and quasi-identities that hold in all CRLs.

$$
\begin{aligned}
& x \approx e \rightarrow x \approx|x| \rightarrow x \approx x \cdot|x| \\
& e \leq|x| \approx\|x\| \\
& e \leq x \Longleftrightarrow|| x \mid \leq x \\
& x \leq y \Longleftrightarrow e \leq x \rightarrow y \Longleftrightarrow|x \rightarrow y| \leq x \rightarrow y \\
& x \rightarrow(y \rightarrow z) \approx(y \cdot x) \rightarrow z, \text { in particular: } \\
& x \rightarrow(x \rightarrow y) \approx x^{2} \rightarrow y \text { and } x \rightarrow|x| \approx x^{2} \rightarrow x \\
& x \rightarrow(y \rightarrow z) \approx y \rightarrow(x \rightarrow z) \\
& x \leq(x \rightarrow y) \rightarrow y \\
& ((x \rightarrow y) \rightarrow y) \rightarrow y \approx x \rightarrow y \\
& |x| \rightarrow y \leq y \\
& (x \vee y) \cdot z \approx(x \cdot z) \vee(y \cdot z)
\end{aligned}
$$

Because CRLs are lattice expansions, the variety of all CRLs is congruence distributive. It is also congruence permutable and has the congruence extension property (see [1, 19]). Recall that a CRL is said to be integral if its identity $e$ is its greatest element, and square increasing if it satisfies $x \leq x^{2}$. It is square decreasing if it satisfies $x^{2} \leq x$. The subdirectly irreducible CRLs are characterized in the next lemma, a proof of which can be found for instance in [10]. (Stronger results of this kind will appear in 34.)

\section{Lemma 3.}

(i) A CRL $\boldsymbol{A}$ is subdirectly irreducible iff there exists an element $b \in A$ such that (I) $b<e$, and (II) whenever $a \in A$ with $a<e$, then there is a positive integer $n$ for which $a^{n} \leq b$. In particular:

(ii) A square increasing CRL $\boldsymbol{A}$ is subdirectly irreducible iff $\{x \in A: x<e\}$ has a greatest element. 
The variety of square increasing CRLs has equationally definable principal congruences (EDPC) in the sense of [8]; this was pointed out in [1]. We shall not discuss EDPC in detail here, since we shall rely only on the following two facts about it. First, it follows immediately from the definition that EDPC persists in subvarieties. Secondly, the following finite basis theorem for varieties with EDPC was proved by Blok and Pigozzi.

Theorem 4 (8]). In a finitely axiomatized variety with EDPC, any subclass axiomatized by a finite number of positive universal sentences generates a finitely axiomatized subvariety.

Representable CRLs were defined in the introduction, and they are obviously always distributive. Every representable CRL is in fact a subdirect product of linear CRLs, because the class of linear CRLs is obviously closed under subalgebras.

A finite equational basis for the variety of all CRLs can be found in [24]. It is observed in 24] that the representable CRLs also form a variety. The proof amounts to showing that this class coincides with the variety $\mathrm{V}$ generated by all linear CRLs. Furthermore, it is shown in 24] that the variety of representable CRLs is axiomatized, relative to the variety of all CRLs, by the identity 1

$$
e \leq(x \rightarrow y) \vee(y \rightarrow x)
$$

Definition 2. We denote by RICRL the class of all representable idempotent CRLs, or briefly, all RICRLs.

By the preceding remarks, RICRL is also a finitely axiomatized variety.

Definition 3. A unary operation $\neg$ on a $\mathrm{CRL} \boldsymbol{A}=\langle A ; \cdot, \rightarrow, \wedge, \vee, e\rangle$ is called an involution if for all $a, b \in A$,

$$
\neg \neg a=a \quad \text { and } \quad a \rightarrow \neg b=b \rightarrow \neg a .
$$

In this case the $\{\neg\}$-expansion $\boldsymbol{A}\urcorner=\langle A ; \cdot, \rightarrow, \wedge, \vee, \neg, e\rangle$ of $\boldsymbol{A}$ is called an involutive $C R L$. We also call $\boldsymbol{A}$ the $C R L$-reduct or the $\{\neg\}$-free reduct of $\boldsymbol{A}\urcorner$.

Every involutive CRL satisfies

$$
\neg x \approx x \rightarrow \neg e .
$$

Thus, an involutive CRL $\boldsymbol{A}\urcorner$ and its $\{\neg\}$-free reduct $\boldsymbol{A}$ have the same congruences. So $\boldsymbol{A}\urcorner$ is subdirectly irreducible iff $\boldsymbol{A}$ is. Note that if we define $f:=\neg e$, then involutive CRLs satisfy

$$
(x \rightarrow f) \rightarrow f \approx x .
$$

Up to term equivalence, the involutive CRLs may be characterized as the expansions of CRLs $\boldsymbol{A}$ by a distinguished element $f \in A$ satisfying (13). For in any such expansion, if we define $\neg x:=x \rightarrow f$, then the algebra satisfies

$$
x \rightarrow \neg y=x \rightarrow(y \rightarrow f)=y \rightarrow(x \rightarrow f)=y \rightarrow \neg x,
$$

as a consequence of (7).

Every involutive CRL satisfies

$$
x \cdot y \approx \neg(x \rightarrow \neg y) \text { and } x \rightarrow y \approx \neg(x \cdot \neg y),
$$

\footnotetext{
${ }^{1}$ In [24, another identity, $e \wedge(x \vee y) \approx(e \wedge x) \vee(e \wedge y)$, is included in the axiomatization. But this axiom can be shown to hold in all CRLs that satisfy (12).
} 
as well as de Morgan's laws for $\neg, \wedge, \vee$. The operation $\neg$ is therefore a lattice anti-isomorphism between the lattice reduct of an involutive CRL and its dual.

An involutive CRL is said to be idempotent, distributive or linear (respectively) if its CRL-reduct has the indicated property. It is said to be representable if it is a subdirect product of linear involutive CRLs or, equivalently, if its CRL-reduct is representable.

The $M V$-algebras of Chang [15] are examples of representable integral (hence square decreasing) involutive CRLs that need not be idempotent. Up to term equivalence, the involution-free subreducts of MV-algebras are just the Wajsberg hoops of [7. The varieties of MV-algebras and of Wajsberg hoops are not locally finite, but it follows from the theory in [7] that every locally finite variety of MValgebras or of Wajsberg hoops is finitely generated.

\section{Brouwerian lattices and relative Stone algebras}

The class of relative Stone algebras and the class of positive Sugihara monoids are two well-understood locally finite varieties of RICRLs that are not finitely generated. These two varieties are incomparable in their extent. Eventually we shall obtain a constructive characterization of all subdirectly irreducible RICRLs (Theorem 201), and it will be helpful to view this as a simultaneous generalization of simpler structure theorems for these subvarieties. Therefore, in this section and the next, we recall descriptions of the subdirectly irreducible algebras in the subvarieties.

Definition 4. An integral idempotent CRL is called a Brouwerian lattice. A representable Brouwerian lattice is called a relative Stone algebra. The class of all relative Stone algebras is denoted by RSA.

Recall from the introduction that every integral CRL is square decreasing; hence every integral square increasing CRL is idempotent and therefore a Brouwerian lattice. Note that RSA is just the class of all integral members of RICRL.

Brouwerian lattices are in fact just the $\{\perp\}$-free subreducts of Heyting algebras. In these algebras the operations $\cdot$ and $\wedge$ coincide, and $\rightarrow$ is relative pseudocomplementation. Since $\cdot$ distributes over $\vee$ in every CRL, it follows that Brouwerian lattices are distributive, although they need not be representable. Clearly, the variety RSA is axiomatized, relative to Brouwerian lattices, by the identity $(x \rightarrow y) \vee(y \rightarrow x) \approx e$. It is well known that the variety of all Brouwerian lattices is not locally finite (see the remarks after Corollary 19).

Example 1. Every chain $\langle A ; \wedge, \vee\rangle$ with a top element $e$ becomes a Brouwerian lattice - in fact a relative Stone algebra $\langle A ; \wedge, \rightarrow, \wedge, \vee, e\rangle$ with the same lattice order $\leq$ if we define, for $a, b \in A$, that $a \rightarrow b=e$ whenever $a \leq b$, and $a \rightarrow b=b$ otherwise. By Lemma 3(ii), this algebra is subdirectly irreducible iff $e$ has a subcover, i.e., iff $\langle A ; \wedge, \vee\rangle$ has a co-atom.

Conversely, since $\cdot$ and $\wedge$ must coincide in Brouwerian lattices, and since every subdirectly irreducible representable CRL is linear, every subdirectly irreducible algebra in RSA arises from a chain with a co-atom in the manner described above.

It follows in particular that an $n$-generated subdirectly irreducible relative Stone algebra has at most $n+1$ elements - the generators and $e$. Since this bound depends only on the number of generators, we infer from Theorem 1 that the variety RSA is locally finite. 


\section{SugIhARA MONOIDS AND THEIR SUBREDUCTS}

Definition 5. An idempotent distributive involutive CRL is called a Sugihara monoid. The class of all Sugihara monoids will be denoted by SM.2

Evidently SM is a variety and we shall see presently that it consists of representable algebras. The following four examples of linear Sugihara monoids turn out to be canonical.

Example 2. Let $Z$ denote the set of all integers and $\leq$ the conventional total order of $Z$. Consider also the nonstandard total order $\preceq$ of $Z$ defined by

$$
\ldots \prec-3 \prec 3 \prec-2 \prec 2 \prec-1 \prec 1 \prec 0,
$$

i.e., numbers are ordered by descending absolute value, but two numbers with the same absolute value are put in the conventional order. Let $\cdot$ denote the binary minimum operation on $Z$ with respect to $\preceq$, so $\cdot$ is associative, commutative and idempotent, with identity 0 . It is easy to see that $\langle Z ; \cdot, \wedge, \vee, 0\rangle$ is a lattice-ordered monoid, where $\wedge$ and $\vee$ are the conventional minimum and maximum operations. We can characterize $\cdot$ without reference to $\preceq$ as follows:

$$
x \cdot y= \begin{cases}x \wedge y & \text { if }|x|=|y|, \\ y & \text { if }|x|<|y|, \\ x & \text { if }|y|<|x| .\end{cases}
$$

Also, $\langle Z ; \cdot, \wedge, \vee, 0\rangle$ is residuated. Its residual operation $\rightarrow$ can be characterized by

$$
x \rightarrow y= \begin{cases}(-x) \vee y & \text { if } x \leq y, \\ (-x) \wedge y & \text { if } x \leq \leq y,\end{cases}
$$

where $-x$ denotes the usual additive inverse of $x$. Now $Z^{\urcorner}=\langle Z ; \cdot, \rightarrow, \wedge, \vee,-, 0\rangle$ is a Sugihara monoid. Observe that in $Z^{\urcorner}$, the term function of $x \rightarrow x$ is the conventional absolute value function, so the notation $|x|$ from Definition 1 is not ambiguous.

Example 3. The subset $Z \backslash\{0\}$ of $Z$ is closed under all operations of $\boldsymbol{Z}\urcorner$ except for the nullary operation 0 , and in fact

$$
Z\urcorner \backslash\{0\}=\langle Z \backslash\{0\} ; \cdot, \rightarrow, \wedge, \vee,-, 1\rangle
$$

is a Sugihara monoid. Note that 1 is the identity element of this algebra.

Example 4. For each nonnegative integer $m$, the set

$$
Z_{2 m+1}=\{-m,-m+1, \ldots,-1,0,1, \ldots, m-1, m\}
$$

is the universe of a subalgebra $\boldsymbol{Z}_{2 m+1}^{\urcorner}$of $\boldsymbol{Z}^{\urcorner}$, so $\boldsymbol{Z}_{2 m+1}^{\urcorner}$is also a Sugihara monoid.

Example 5. For each positive integer $m$, the set

$$
Z_{2 m}=\{-m,-m+1, \ldots,-1,1, \ldots, m-1, m\}
$$

is the universe of a subalgebra $\boldsymbol{Z}_{2 m}^{\urcorner}$of $\boldsymbol{Z}^{\urcorner} \backslash\{0\}$, so $\boldsymbol{Z}_{2 m}^{\urcorner}$is a Sugihara monoid.

By Lemma 3(ii), all the algebras defined in Examples 25 are subdirectly irreducible, except for the trivial $\boldsymbol{Z}_{1}$. The following partial converse of this statement is due to Dunn. It was inspired by an earlier result of Meyer [29].

\footnotetext{
${ }^{2}$ The 'Sugihara algebras' studied for instance in [17 2, 6, 11, may be characterized as the $\{e\}$-free subreducts of Sugihara monoids, but these algebras shall not concern us here. The name comes from 40].
} 
Theorem 5 ([2, pp. 422-423]; see [17] also).

(i) Sugihara monoids are representable.

(ii) Up to isomorphism, the finite subdirectly irreducible Sugihara monoids are just the algebras $\boldsymbol{Z}_{n}^{\urcorner}, n>1$.

More exactly, Dunn proved in 2] that any subdirectly irreducible Sugihara monoid is linear and satisfies (14). Taking into account that • is definable in terms of $\neg$ and $\rightarrow$, we can deduce that every $n$-generated subdirectly irreducible Sugihara monoid has at most $2 n+2$ elements - the generators and their involutive images, $e$ and $\neg e$. So by Theorem 1 the variety of Sugihara monoids is locally finite.

Definition 6. A CRL is called a positive Sugihara monoid, or a PSM if it can be embedded into (the CRL-reduct of) some Sugihara monoid. The class of all such algebras will be denoted by PSM.

In other words, PSMs are just the $\{\cdot, \rightarrow, \wedge, \vee, e\}$-subreducts of Sugihara monoids. In [35] it is proved that the class PSM is a variety. It is locally finite, since SM is locally finite. In Section 9 we shall establish an equational basis for PSM (Theorem 23).

Definition 7. The CRL-reduct $\langle Z ; \cdot, \rightarrow, \wedge, \vee, 0\rangle$ of the Sugihara monoid $\boldsymbol{Z}\urcorner$ (see Example 21) will be denoted by $\boldsymbol{Z}$. Similarly, $\boldsymbol{Z} \backslash\{0\}$ and $\boldsymbol{Z}_{n}$ shall denote the CRL-reducts of $\boldsymbol{Z}\urcorner \backslash\{0\}$ and of $\boldsymbol{Z}_{n}^{\urcorner}, n>0$, respectively.

The next result is a consequence of Theorem 5 and local finiteness. Again, a proof can be found in [35.

Corollary 6. Up to isomorphism, the finite subdirectly irreducible positive Sugihara monoids are just the algebras $\boldsymbol{Z}_{n}, n>1$.

Note in particular that positive Sugihara monoids are also representable, i.e., $\mathrm{PSM} \subseteq \mathrm{RICRL}$.

The subvarieties RSA and PSM of RICRL are incomparable in their extent, since $\boldsymbol{Z}_{3} \in \mathrm{PSM}$ is not integral, while the linear relative Stone algebra constructed from a three-element chain as in Example 1 is subdirectly irreducible but it is not among the algebras $\boldsymbol{Z}_{n}, n>1$. In fact, RSA $\cap$ PSM is just the variety of (dual) generalized Boolean algebras of [5], which is generated by $\boldsymbol{Z}_{2}$. Galatos [21] has shown that $\boldsymbol{Z}_{2}$ and $\boldsymbol{Z}_{3}$ generate the only two minimal varieties of idempotent CRLs.

We have seen that every subdirectly irreducible $n$-generated algebra in RSA or in PSM has at most $2 n+2$ elements. So, if the varietal join of RSA and PSM were equal to RICRL, then RICRL would be locally finite, in view of the congruence distributivity of CRLs and Theorems2(iii) and1, The next example shows, however, that RICRL is strictly more inclusive than this join.

Example 6. Let $E=\{-3,0,1,2,3\}$ and note that $E$ is a subuniverse of the $\{\cdot \wedge, \vee, e\}$-reduct of $\boldsymbol{Z}$ (where $e=0$ ). If $a, b \in E$, then $a \cdot-3 \leq 3 \cdot-3=-3 \leq b$, so there is always a greatest $c \in E$ such that $a \cdot c \leq b$. The lattice-ordered commutative monoid $\langle E ; \cdot, \wedge, \vee, 0\rangle$ is therefore residuated. Let $\rightarrow$ denote its residuation operation, which differs from that of $\boldsymbol{Z}$, e.g., we now have $2 \rightarrow 1=-3 \neq(-2) \wedge 1$. Then $\boldsymbol{E}=\langle E ; \cdot, \rightarrow, \wedge, \vee, 0\rangle \in \mathrm{RICRL}$ and $\boldsymbol{E}$ is subdirectly irreducible, by Lemma 3(iii).

Let $V$ denote the join HSP(RSA $\cup$ PSM) of the varieties RSA and PSM. Since $V$ is congruence distributive, Theorem 2(ii) gives

$$
\mathrm{V}_{\mathrm{SI}}=\mathrm{RSA}_{\mathrm{SI}} \cup \mathrm{PSM}_{\mathrm{SI}} \text {. }
$$


Obviously, $\boldsymbol{E} \notin \mathrm{RSA}$ SI , as $\boldsymbol{E}$ is not integral. Also $|E|=5$ but $\boldsymbol{E} ¥ \boldsymbol{Z}_{5}$, since the identity is an atom of $\boldsymbol{E}$ but not of $\boldsymbol{Z}_{5}$. By Corollary 6 , $\boldsymbol{Z}_{5}$ is the only 5-element subdirectly irreducible PSM (up to isomorphism), so $\boldsymbol{E} \notin \mathrm{PSM}_{\mathrm{SI}}$. This shows that $\boldsymbol{E} \notin \mathrm{V}_{\mathrm{SI}}$. Because $\boldsymbol{E}$ is subdirectly irreducible, it follows that $\boldsymbol{E} \notin \mathrm{V}$. Thus, $\mathrm{V}$ is a proper subvariety of RICRL.

Nevertheless, we shall proceed to prove that RICRL is locally finite. Our strategy will be to show first that every subdirectly irreducible RICRL $\boldsymbol{A}$ satisfies $\forall x \forall y(x \cdot y \approx x$ or $x \cdot y \approx y)$. Thus any $n$-element subset of $A$ is closed under $\cdot$. It is also closed under $\wedge$ and $\vee$ because it is totally ordered. We shall then show that by adding at most $2 n+1$ additional elements, we get a subset closed under $\rightarrow$ as well. Thus we shall conclude that every subdirectly irreducible $n$-generated RICRL has at most $3 n+1$ elements. Again, because this finite bound depends on $n$ alone (and not on the choice of $\boldsymbol{A}$ ), Theorem 1 will allow us to infer that RICRL is locally finite. The next two sections contain the necessary preparations for this strategy.

\section{Square-Tonic CRLs}

Recall that a CRL is said to be square decreasing if it satisfies $x^{2} \leq x$. We shall frequently abbreviate $x \cdot y$ as $x y$.

Proposition 7. Let $\boldsymbol{A}$ be a square decreasing CRL and let $x, y \in A$.

(i) $x \leq|x|$ whence:

(ii) $e \leq x$ iff $|x|=x$.

(iii) $x y \leq x \vee y$, whence:

(iv) if $e \leq x \leq y$, then $x y=y$.

(v) $x \leq|x \rightarrow e|$.

(vi) $|x|(|x| \rightarrow e)=|x| \rightarrow e$.

Proof. From the square decreasing law $x x \leq x$ and the definition of residuation, we infer $x \leq x \rightarrow x(=|x|)$, proving (ii). Then (iii) follows from (ii) and property (3) of Section 3 For (iii), recall that • is order preserving in both arguments, so $x y \leq(x \vee y)(x \vee y) \leq x \vee y$ (by the square decreasing law). Thus, in (iv), if $e \leq x \leq y$, we have $x y \leq x \vee y=y=e y \leq x y$, so $x y=y$.

(v) Every CRL satisfies $x(x \rightarrow e) \leq e$, by definition of residuation. This together with associativity, commutativity and the square decreasing law gives

$$
x((x \rightarrow e) x)=x^{2}(x \rightarrow e) \leq x(x \rightarrow e) \leq e .
$$

Then by the definition of residuation, $(x \rightarrow e) x \leq x \rightarrow e$. By residuation again, $x \leq(x \rightarrow e) \rightarrow(x \rightarrow e)=|x \rightarrow e|$.

(vi) Substituting $|x|$ for $x$ in (吾), we get $|x| \leq(|x| \rightarrow e) \rightarrow(|x| \rightarrow e)$. Then by residuation and commutativity, $|x|(|x| \rightarrow e) \leq|x| \rightarrow e$. For the reverse inequality, we recall that $e \leq|x|$, so

$$
|x| \rightarrow e=e(|x| \rightarrow e) \leq|x|(|x| \rightarrow e),
$$

as required.

We now consider properties of square increasing CRLs, i.e., CRLs that satisfy $x \leq x^{2}$. 
Proposition 8. Let $\boldsymbol{A}$ be a square increasing $C R L$ and let $x, y \in A$.

(i) $x \wedge y \leq x y$.

(ii) If $x \leq y \leq e$, then $|y| \leq|x|$ and $x y=x$.

(iii) If $x \leq e$, then ||$x|\rightarrow e| \leq|x|$.

(iv) If $\boldsymbol{A}$ is linear and $x, y \leq e$ and $|x|<|y|$, then $x y=y$.

Proof. For (ii), we use the square increasing law and the isotonicity of $\cdot$ in both arguments to compute that $x \wedge y \leq(x \wedge y)(x \wedge y) \leq x y$.

(iii) Let $x \leq y \leq e$. Since $e \leq|x|$, we have $y \leq|x|$. By monotonicity properties of $\rightarrow$ and (6),

$$
y \rightarrow y \leq x \rightarrow y \leq x \rightarrow|x|=x^{2} \rightarrow x \leq x \rightarrow x
$$

(where the last inequality relies on the square increasing law), i.e., $|y| \leq|x|$. Also, using (1), we compute that $x=x \wedge y \leq x y \leq x e=x$, so $x y=x$.

(iii) Let $x \leq e$ and define $z:=|x| \rightarrow e$. We must show that $|z| \leq|x|$. Now $|x| x=x$ (by (11) and commutativity), so $|x| x \leq e$. By residuation, $x \leq|x| \rightarrow e$, i.e., $x \leq z$. Also, $|x| \rightarrow e \leq e$, by (10), so $x \leq z \leq e$. Then by (iii), $|z| \leq|x|$.

(iv) Let $\boldsymbol{A}$ be linear and assume that $x, y \leq e$ and $|x|<|y|$. By (iii), $x \not \leq y$. By linearity, therefore, $y<x \leq e$. Then by (iii), $x y=y$.

Corollary 9. Let $\boldsymbol{A}$ be a square increasing $C R L$ and let $e \leq z \in A$. Then $\{x \in$ $A: x<e$ and $|x|=z\}$ is an interval of $\langle A ; \leq\rangle$.

Proof. Suppose $x \leq w \leq y<e$, with $|x|=|y|=z$. By Proposition 8(iii), we have $z=|y| \leq|w| \leq|x|=z$, so $|w|=z$, as required.

\section{IDEMPOTENCE}

We derive some properties of idempotent CRLs, i.e., ones satisfying $x^{2} \approx x$.

Proposition 10. Let $\boldsymbol{A}$ be an idempotent $C R L$ and let $x, y \in A$.

(i) $x \rightarrow|x|=|x|$.

(ii) If $x \leq y$, then $x \leq x y \leq y$.

(iii) If $y \leq x$ and $|x| \leq|y|$, then $x y=y$.

(iv) If $|x|=|y|$, then $x y=x \wedge y$.

(v) If $x \leq e$, then $\| x|\rightarrow e|=|x|$.

Proof. (1i) By (6) and idempotence, $x \rightarrow|x|=x^{2} \rightarrow x=x \rightarrow x=|x|$.

(iii) If $x \leq y$, then, by Propositions 7(iii) and8(8i), $x=x \wedge y \leq x y \leq x \vee y=y$.

(iii) Let $y \leq x$ and $|x| \leq|y|$. By (iii), $y \leq y x$. Also, by Proposition 7(ii), $x \leq|x| \leq|y|=y \rightarrow y$, so $y x \leq y$, by residuation. Therefore, $y x=y$, i.e., $x y=y$ (by commutativity).

(iv) By Proposition 8(1), $x \wedge y \leq x y$. By Proposition 7(1), $z \leq|z|$ for all $z \in A$. So $x y \leq x|y|=x|x|=x$, by (11). In view of commutativity, it follows similarly that $x y \leq y$. So $x y \leq x \wedge y$; therefore $x y=x \wedge y$.

(vi) follows from Propositions 7(v) (substitute $|x|$ for $x$ ) and 8(iii).

Every subdirectly irreducible RICRL is linear, so from this point onward, we shall focus on properties of linear idempotent CRLs.

Proposition 11. Let $\boldsymbol{A}$ be a linear idempotent $C R L$ and let $x, y \in A$. If $x \leq e \leq$ $y$ and $|x|<|y|$, then $x y=y$. 
Proof. Assume that $x \leq e \leq y$ and $|x|<|y|$, so $|y|=y$, by Proposition 7 (iii). By Proposition 10(ii), $x y \leq y$. We claim that $x y \not \leq e$. Suppose, on the contrary, that $x y \leq e$. Then $x y \leq|x|$, because $e \leq|x|$. By residuation, $y \leq x \rightarrow|x|$. By Proposition 10(i), $x \rightarrow|x|=|x|<|y|=y$, so $y<y$, a contradiction. Therefore, $x y \not \leq e$, whence $e<x y$ (by linearity). Now, by the isotonicity, associativity and idempotence of $\cdot$,

$$
y=e y \leq(x y) y=x y^{2}=x y,
$$

so $x y=y$.

Theorem 12. Let $\boldsymbol{A}$ be an idempotent linear $C R L$ and let $x, y \in A$.

(i) If $|x|=|y|$, then $x y=x \wedge y \in\{x, y\}$.

(ii) If $|x|<|y|$, then $x y=y$. Consequently:

(iii) $\boldsymbol{A}$ satisfies $\forall x \forall y(x y \approx x$ or $x y \approx y)$.

Proof. (ii) follows from Proposition 10(iv), by linearity.

(ii) By Proposition 10(iii), we may assume without loss of generality that $y \not \leq x$, so let $x<y$. Now the result follows from Propositions 7(iv), (8) and 11. Clearly, (iii) follows from (ii) and (iii).

This accomplishes the first task in the strategy laid out at the end of Section 5 for proving local finiteness of RICRL. We begin the second task, which is to show that any $n$-element subset of an idempotent linear CRL can be extended to a $(3 n+1)$-element subalgebra. Theorem 12 and linearity show that we need only concern ourselves with closure under $\rightarrow$.

Proposition 13. Let $\boldsymbol{A}$ be an idempotent linear CRL and let $x \in A$. If $x \leq e$, then $(|x| \rightarrow e) \rightarrow e=|x|$.

Proof. Define $y:=|x| \rightarrow e$, so $y \leq e$, by (10). We must show that $y \rightarrow e=|x|$. By Propositions 10(v) and 7)(vi), $|y|=|x|$ and $|x| y=y$ so, by commutativity, $y|x|=y \leq e$.

To establish that $y \rightarrow e=|x|$, it is enough, by the definition of residuation, to show that whenever $z \in A$ with $z \not \leq|x|$, then $y z \not \leq e$. Suppose $z$ is as just described. By linearity, $|x|<z$, so $e<z$, whence $z=|z|$ (see (2) and Proposition 7(1ii)). Now $|y|=|x|<z=|z|$, so by Theorem 12(ii), $y z=z$. But $e<z$ so $y z \not \leq e$, as required. Thus, $|x|=y \rightarrow e$.

We may now characterize residuation in idempotent linear CRLs in a simple manner. The next result should be compared with the characterization of $\rightarrow$ in the Sugihara monoid $Z^{\urcorner}$, given in (14).

Theorem 14. Let $\boldsymbol{A}$ be an idempotent linear $C R L$ and let $x, y \in A$.

(i) If $x \leq y$, then $x \rightarrow y=|x| \vee y$.

(ii) If $x>y$, then $x \rightarrow y=(x \rightarrow e) \wedge y$.

Proof. (ii) Let $x \leq y$. Then $x y \leq y$, by idempotence. By (11) and (11),

$$
x(|x| \vee y)=x|x| \vee x y=x \vee x y \leq y .
$$

If $z \in A$ and $z \not \leq|x| \vee y$, then $|x| \vee y<z$ (by linearity) so $y<z$ and $e \leq|x|<z$, whence $|z|=z$. Thus, $|x|<|z|$. Then by Theorem 12(ii), $x z=z \not \leq y$. This shows that $|x| \vee y$ is the largest $z \in A$ such that $x z \leq y$, i.e., $|x| \vee y=x \rightarrow y$. 
(iii) Let $x>y$. Since $\boldsymbol{A}$ is linear and - preserves order,

$$
x((x \rightarrow e) \wedge y)=x(x \rightarrow e) \wedge x y .
$$

First we shall show that $x(x \rightarrow e) \wedge x y \leq y$. Suppose this is not the case. Then by linearity, $x(x \rightarrow e) \wedge x y>y$, so

$$
x(x \rightarrow e)>y \text { and } x y>y .
$$

From $x y>y$ and Theorem 12, we infer that $x y=x$ and that $|y|<|x|$. It follows that we cannot have $y<x \leq e$ (see Proposition 8(iii)), so $e<x$, whence $x=|x|$. Because $x y=x \not \leq e$, the definition of residuation gives $y \leq x x \rightarrow e$, so $x \rightarrow e<y<x$. We also have $y<x(x \rightarrow e) \leq e$ (from (15D)), so $y<e$.

We cannot have $x<|x \rightarrow e|$, because Theorem 12(ii) would then yield $x(x \rightarrow e)=x \rightarrow e<y$, a contradiction. So $|x \rightarrow e| \leq x$. By Proposition 7(v), $x=|x \rightarrow e|$. Now by Theorem 12(1), $x(x \rightarrow e)=x \wedge(x \rightarrow e)=x \rightarrow e<y$, a contradiction. This shows that $x((x \rightarrow e) \wedge y) \leq y$.

Now suppose $z \in A$ with $x z \leq y$. If we can show that $z \leq(x \rightarrow e) \wedge y$, it will follow that $(x \rightarrow e) \wedge y=x \rightarrow y$, by definition of residuation. We proceed to do this.

Since $x z \leq y$, we have $x z \neq x$, so $x z=z$, by Theorem 12(iii), so $z \leq y$. It remains only to show that $z \leq x \rightarrow e$, i.e., that $x z \leq e$, i.e., that $z \leq e$. Suppose, on the contrary, that $z \not \leq e$. By linearity, $e<z$. Then $e<x$, because $z \leq y<x$. So $|z|=z$ and $|x|=x$. In particular, $|z|<|x|$, so by Theorem [12(ii), $x z=x$, a contradiction. Thus, $z \leq x \rightarrow e$, which completes the proof of (iii).

Definition 8. We define unary terms $\alpha(x):=|x|$ and $\beta(x):=x \rightarrow e$.

Note that all CRLs satisfy $\alpha \alpha(x) \approx \alpha(x)$ and $\beta \beta \beta(x) \approx \beta(x)$, by (11) and (9). Theorem 14 simplifies our task in the following way:

Corollary 15. Let $W$ be a subset of an idempotent linear CRL $\boldsymbol{A}$. If $e \in W$ and $W$ is closed under $\alpha$ and $\beta$, then $W$ is a subuniverse of $\boldsymbol{A}$.

Proof. This follows from linearity and Theorems 12(iii) and 14.

When employing this corollary, we will need to consider terms such as $\alpha \beta(x)$ and $\beta \alpha(x)$. The next result will help us to deal with these.

Corollary 16. Let $\boldsymbol{A}$ be an idempotent linear $C R L$ and let $e \leq y \in A$. Then:

(i) $(y \rightarrow e) \rightarrow e=|y \rightarrow e|$;

(ii) $|y \rightarrow e| \rightarrow e=y \rightarrow e$.

Proof. We have $y \rightarrow e \leq e \rightarrow e=e$. Now:

(ii) $(y \rightarrow e) \rightarrow e=|y \rightarrow e| \vee e$ (by Theorem 14(1i)) $=|y \rightarrow e|$, so:

(iii) $|y \rightarrow e| \rightarrow e=((y \rightarrow e) \rightarrow e) \rightarrow e=y \rightarrow e$ (by (9) ) ).

\section{The MAin Results}

In an algebra $\boldsymbol{A}$, the subalgebra generated by a subset $X$ of $A$ will be denoted by $S g^{\boldsymbol{A}}(X)$.

Theorem 17. Let $\boldsymbol{A}$ be an $n$-generated subdirectly irreducible RICRL, where $n$ is finite. Then $|A| \leq 3 n+1$. 


$$
\begin{aligned}
& \left|x_{i}\right| \\
& \left|y_{j} \rightarrow e\right| \\
& y_{j} \\
& e \\
& y_{j} \rightarrow e \\
& \left|x_{i}\right| \rightarrow e \\
& x_{i}
\end{aligned}
$$

Figure 1. Example of $W$ in the 2-generated case where $X=$ $\left\{x_{i}, y_{j}\right\}$ and $y_{j}<\left|x_{i}\right|$

Proof. Clearly $\boldsymbol{A}$ is an idempotent linear CRL. Let $X \subseteq A$ with $|X| \leq n$, such that $S g^{\boldsymbol{A}}(X)=A$. Without loss of generality, assume that $e \notin X$. Let

$$
X=\left\{x_{0}, \ldots, x_{m-1}\right\} \cup\left\{y_{0}, \ldots, y_{r-1}\right\},
$$

where $x_{i}<e<y_{j}$ for all $i<m$ and all $j<r$, so $m+r \leq n$. Define

$$
\begin{array}{r}
W:=\{e\} \cup X \cup\left\{\left|x_{i}\right|: i<m\right\} \cup\left\{\left|x_{i}\right| \rightarrow e: i<m\right\} \\
\cup\left\{y_{j} \rightarrow e: j<r\right\} \cup\left\{\left|y_{j} \rightarrow e\right|: j<r\right\} .
\end{array}
$$

Then $|W| \leq 1+n+2 m+2 r \leq 3 n+1$. (See Figure 1.) Now $W$ is closed under $\alpha$ and $\beta$ (see Definition [8), because $\alpha$ is idempotent over $\boldsymbol{A}$ and $|e|=e \rightarrow e=e$ and

$$
\begin{array}{ll}
x_{i} \rightarrow e=\left|x_{i}\right| \vee e=\left|x_{i}\right| & \text { (Theorem 14(11)) } \\
\left|y_{j}\right|=y_{j} & \text { (Proposition [7(1ii)) } \\
|| x_{i}|\rightarrow e|=\left|x_{i}\right| & \text { (Proposition 10((v)) } \\
\left(\left|x_{i}\right| \rightarrow e\right) \rightarrow e=\left|x_{i}\right| & \text { (Proposition 13) } \\
\left(y_{j} \rightarrow e\right) \rightarrow e=\left|y_{j} \rightarrow e\right| & \text { (Corollary 16(1i)) } \\
\left|y_{j} \rightarrow e\right| \rightarrow e=y_{j} \rightarrow e & \text { (Corollary [16(1ii)). }
\end{array}
$$

Since $\{e\} \cup X \subseteq W$, it follows from Corollary 15 that

$$
W=S g^{\boldsymbol{A}}(W) \supseteq S g^{\boldsymbol{A}}(X)=A,
$$

so $A=W$, whence $|A| \leq 3 n+1$. 
As the next example shows, the bound in Theorem 17 is attained when $n=1$, so we cannot replace $\leq$ by $<$ in its statement.

Example 7. Recall the algebra $\boldsymbol{E}$ of Example 6. The subalgebra $\boldsymbol{C}$ of $\boldsymbol{E}$ with universe $C=\{-3,0,2,3\}$ is generated by $\{2\}$. It is an idempotent linear CRL that is subdirectly irreducible, by Lemma 3(ii), so it is a 1-generated member of $\mathrm{RICRL}_{\mathrm{SI}}$, and $|C|=4=3 \cdot 1+1$.

From Theorems 1 and 17, we conclude:

Theorem 18. The variety RICRL is locally finite.

Corollary 19. Every finitely axiomatized subquasivariety of RICRL has a decidable quasi-equational theory.

It seems that within the context of CRLs, we cannot generalize Theorem 18 by relaxing its hypotheses in any natural way. In particular, 'representable' cannot be weakened to 'distributive', as the idempotent distributive CRLs include all Brouwerian lattices and this subvariety is not locally finite. Indeed, Rieger 38 and Nishimura 33. showed independently that the free 1-generated Heyting algebra is infinite. Its $\{\perp\}$-free reduct is therefore an infinite 2-generated Brouwerian lattice. See [5, Ch. IX] for the details. Also, we cannot weaken 'idempotent' to 'square decreasing' nor to 'square increasing'. To see this, note that the infinite naturally ordered additive monoid of nonpositive integers is square decreasing, residuated, representable and generated as a CRL by $\{-1\}$, while the infinite conventionally ordered multiplicative monoid on $\{0\} \cup\left\{2^{n}: n \in \omega\right\} \cup\{\infty\}$, with $0 \cdot \infty=\infty \cdot 0=0$ and $x \cdot \infty=\infty \cdot x=\infty$ for $x \neq 0$, is square increasing, residuated, representable and generated as a CRL by $\{2\}$. Finally, we cannot weaken 'idempotent' to 'satisfying $x^{2} \approx x^{3}$, as there are infinite representable integral CRLs with this property that are finitely generated; this follows from an argument of Dyrda [18] concerning BCK-algebras.

The following constructive characterization of RICRL It will allow us to view any algebra in this class as, roughly speaking, an amalgam of a Sugihara monoid and a family of relative Stone algebras.

Theorem 20. Let $\boldsymbol{A}=\langle A ; \cdot, \rightarrow, \wedge, \vee, e\rangle$ be an algebra of type $\langle 2,2,2,2,0\rangle$ whose $\{\wedge, \vee\}-$ reduct is a totally ordered lattice $\langle A ; \leq\rangle$. The following conditions are equivalent:

(I) $\boldsymbol{A}$ is a subdirectly irreducible idempotent linear $C R L$, i.e., $\boldsymbol{A} \in \mathrm{RICRL}_{\mathrm{SI}}$;

(II) There exists a chain $\left\langle Y ; \leq_{Y}\right\rangle$ with least element $e$ and, for each $y \in Y$, there exists a (possibly empty) chain $\left\langle A_{y} ; \leq_{y}\right\rangle$ such that $Y, A_{y}, A_{z}(y \neq z \in Y)$ are mutually disjoint and

(i) $A=Y \cup\left(\bigcup_{y \in Y} A_{y}\right)$ and $\leq$ extends $\leq_{Y} \cup\left(\bigcup_{y \in Y} \leq_{y}\right)$;

(ii) if $y, z, w \in Y$ and $y<_{Y} z$ and $a_{y} \in A_{y}$ and $a_{z} \in A_{z}$, then $a_{z}<a_{y}<w$;

(iii) if $y \in Y$ and $A_{y} \neq \emptyset$, then $A_{y}$ has a greatest element, denoted by $y^{\prime}$;

(iv) if $y \in Y$ and $A_{y}=\emptyset$, then $\left\{z \in Y: y<z\right.$ and $\left.A_{z} \neq \emptyset\right\}$ has a least element, denoted by $\uparrow y$; 
$\begin{cases}z & =\uparrow y=z^{*}=z^{\prime *}=-\left(z^{\prime}\right)=\left|z^{\prime}\right| \\ y & =y^{*}\left(\text { with } A_{y}=\emptyset\right) \\ w & =w^{*}=w^{\prime *}=-\left(w^{\prime}\right)=\left|w^{\prime}\right| \\ e & =e^{*}=-\left(e^{\prime}\right)=e^{\prime *}=\left|e^{\prime}\right| \\ e^{\prime} & =-e \\ A_{e} & =-w \\ A_{z} & =-z=-y\end{cases}$

Figure 2. Example of $\boldsymbol{A} \in \mathrm{RICRL}_{\mathrm{SI}}$ illustrating Theorem 20, with $A_{e} \neq \emptyset$

and if we define unary functions * and - on $A$ by

$$
\left\{\begin{array} { l l } 
{ y ^ { * } = y } & { \text { if } y \in Y } \\
{ a _ { y } ^ { * } = y } & { \text { if } y \in Y , a _ { y } \in A _ { y } }
\end{array} \quad \left\{\begin{array}{ll}
-e=e & \text { if } A_{e}=\emptyset \\
-x=(\uparrow x)^{\prime} & \text { if } e \neq x \in Y, A_{x}=\emptyset \\
-x=x^{\prime} & \text { if } x \in Y, A_{x} \neq \emptyset \\
-x=x^{*} & \text { if } x \notin Y,
\end{array}\right.\right.
$$

then for any $u, v \in A$,

(v) $u \cdot v= \begin{cases}u \wedge v & \text { if } u^{*}=v^{*}, \\ v & \text { if } u^{*}<v^{*}, \\ u & \text { if } v^{*}<u^{*},\end{cases}$

(vi) $u \rightarrow v=\left\{\begin{array}{ll}u^{*} \vee v & \text { if } u \leq v, \\ (-u) \wedge v & \text { if } u>v .\end{array} \quad\right.$ (Condition (II) ends here.)

In this case, we must have $u^{*}=u \rightarrow u$ and $-u=u \rightarrow-e$ for all $u \in A$, and if $A_{e} \neq \emptyset$, then $-e$ is the subcover of $e$.

Proof. (I) $\Rightarrow$ (II). Assume (I) and define

$$
Y=\{a \in A: e \leq a\} .
$$

Let $\leq_{Y}$ be the restriction of $\leq$ to $Y$. For each $y \in Y$, define

$$
A_{y}:=\{c \in A: c<e \text { and }|c|=y\}
$$


and let $\leq_{y}$ be the restriction of $\leq$ to $A_{y}$. By (2) and Proposition 7(1ii), $A$ is the disjoint union of $Y$ and the sets $A_{y}$, so (ii) holds. By Corollary 9, each $A_{y}$ is an interval of $\langle A ; \leq\rangle$, and (iii) follows from Proposition 8)(ii).

Since $\boldsymbol{A}$ is subdirectly irreducible and square increasing, $e$ has a subcover in $\langle A ; \leq\rangle$, by Lemma 3(ii). Let $b$ denote this subcover of $e$.

To verify (iii), consider any $y \in Y$. Suppose $A_{y} \neq \emptyset$, so $y=|x|$ for some $x<e$, i.e., for some $x \in A_{y}$.

Consider first the case $y=e$. We claim that $b$ belongs to $A_{e}$, in which case $b$ is obviously the greatest element of $A_{e}$. Certainly, $b<e$. Since $x<e$, we have $x \leq b$, so, by Proposition 8(iii), $|b| \leq|x|=e$, because $x \in A_{e}$. It follows that $|b|=e$, i.e., $b \in A_{e}$, as required.

Now consider the case where $y \neq e$, i.e., $e<y$. In this case $e \not \leq y \rightarrow e$, by (4), so $y \rightarrow e<e$, by linearity. Also, $|y \rightarrow e|=y$, by Proposition 10(可), so $y \rightarrow e \in A_{y}$. To see that $y \rightarrow e$ is the greatest element of $A_{y}$, suppose $c \in A_{y}$. Then $c<e<y$ and $|c|=y=|y|$. By Theorem 12(1), $y c=y \wedge c=c$; hence $y c<e$, so $c \leq y \rightarrow e$, as required. This completes the proof of (iii).

To prove (iv), suppose $A_{y}=\emptyset$.

Again we consider first the case $y=e$. Since $A_{e}=\emptyset$, we have $|b| \notin A_{e}$, i.e., $|b|>e$. Also $A_{|b|} \neq \emptyset$, because $b \in A_{|b|}$. We claim that

$$
|b|=\min _{\leq}\left\{z \in Y: e<z \text { and } A_{z} \neq \emptyset\right\} .
$$

To see this, suppose $|b|>c>e$. If $w \in A_{c}$, then $w<e$, so $w \leq b$. Then by Proposition 80(ii), $|b| \leq|w|=c$, a contradiction. Thus, $A_{c}=\emptyset$, which vindicates the previous claim.

Now consider the case $y \neq e$, i.e., $y>e$. As in the proof of (iii), it follows from linearity that $y \rightarrow e<e$. We have $|y \rightarrow e| \in Y$ and $y \rightarrow e \in A_{|y \rightarrow e|}$ and $y \leq|y \rightarrow e|$ (Proposition $7((\mathbf{v}))$. We cannot have $y=|y \rightarrow e|$, because this would yield the contradiction $y \rightarrow e \in A_{y}=\emptyset$. Therefore, $y<|y \rightarrow e|$. We claim that

$$
|y \rightarrow e|=\min _{\leq}\left\{z \in Y: y<z \text { and } A_{z} \neq \emptyset\right\} .
$$

To see this, let $y<z<|y \rightarrow e|$ and suppose that $A_{z} \neq \emptyset$, say $w \in A_{z}$. Then $w<e$ and $|w|=z>y=|y|$, so $y w=w<e$, by Theorem 12(ii). Now $w \leq y \rightarrow e<e$, so $|y \rightarrow e| \leq|w|$, by Proposition [8(ii), i.e., $|y \rightarrow e| \leq z$, a contradiction. Thus $A_{z}=\emptyset$ and the claim is true.

This completes the proof of (iv). The remaining claims follow from Theorems 12 and 14 .

(II) $\Rightarrow$ (I). Assume (II). Let $\preceq$ be the total order of $A$ such that

$$
\begin{aligned}
& \text { if } u, v \in A \text { and } u^{*}<v^{*}, \text { then } v \prec u \text {; } \\
& \text { if } u, v \in A \text { and } u^{*}=v^{*} \text {, then }[u \preceq v \text { iff } u \leq v] .
\end{aligned}
$$

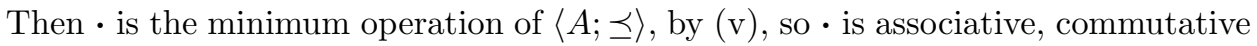
and idempotent on $A$, and $e$ is the identity for $\cdot$. It follows from (者) that • is order preserving in both coordinates with respect to $\leq$, so $\langle A ; \cdot, \wedge, \vee, e\rangle$ is a (linear) lattice-ordered monoid. If $A_{e} \neq \emptyset$, then, by (iii), $e$ has a subcover, viz. $e^{\prime}$. If $A_{e}=\emptyset$, then $(\uparrow e)^{\prime}$ is a subcover of $e$. It follows from (v), (vi) and the definitions that $\langle A ; \cdot, \leq\rangle$ is residuated by $\rightarrow$, so $\boldsymbol{A}$ is an idempotent linear CRL, which is subdirectly irreducible, by Lemma 3(ii). 
Note that the subdirectly irreducible relative Stone algebras are exactly the algebras $\boldsymbol{A}$ as in Theorem 20 that have $Y=\{e\}$ and $A=A_{e} \cup\{e\}$. In a subdirectly irreducible positive Sugihara monoid $\boldsymbol{A}$, we have $\left|A_{y}\right|=1$ for all $y \in Y$, except that $A_{e}$ may be empty. These observations have the following approximate 'converse':

Theorem 21. Let $\boldsymbol{A}$ be a subdirectly irreducible idempotent linear CRL. Let $Y=$ $\{y \in A: e \leq y\}$ and for each $y \in Y$, let $A_{y}=\{c \in A: c<e$ and $|c|=y\}$. Let be defined as in Theorem 20.

(i) For each $y \in Y$, the set $A_{y} \cup\{y\}$ is a subuniverse of the $\{e\}$-free reduct of $\boldsymbol{A}$, and $\boldsymbol{A}(y):=\left\langle A_{y} \cup\{y\} ; \cdot, \rightarrow, \wedge, \vee, y\right\rangle$ is a relative Stone algebra.

(ii) $A_{\text {Sug }}:=\{e,-e\} \cup \bigcup\left\{\{y,-y\}: y \in Y\right.$ and $\left.\left|A_{y}\right|=1\right\}$ is a subuniverse of $\boldsymbol{A}$ which is closed under -, and $\boldsymbol{A}_{\text {Sug }}:=\left\langle A_{\text {Sug }} ; \cdot, \rightarrow, \wedge, \vee,-, e\right\rangle$ is a Sugihara monoid. Consequently:

(iii) If $\left|A_{y}\right| \leq 1$ for all $y \in Y$, and $A_{y} \neq \emptyset$ for all $y \neq e$, then $\boldsymbol{A}$ is a positive Sugihara monoid and the $\{-\}$-expansion of $\boldsymbol{A}$ is a Sugihara monoid.

Proof. It follows easily from Theorem[20(v), (vi) that $A_{y} \cup\{y\}$ and $A_{S u g}$ are closed under the operations in question, and that $y$ is an identity for • on $A_{y} \cup\{y\}$. Since $y$ dominates all elements of $A_{y}$, the algebra $\boldsymbol{A}(y)$ is an integral linear idempotent CRL, i.e., a relative Stone algebra, proving (1). Theorem 20 also shows that $--a=$ $a$ and $a \rightarrow-e=-a$ for all $a \in A_{\text {Sug }}$. So, by the remarks following Definition 3 in Section 3, $\boldsymbol{A}_{S u g}$ is an involutive CRL, and since it is idempotent and linear, it is a Sugihara monoid. Under the assumptions of (iii), we have $A=A_{\text {Sug }}$, so (iii) follows from (iii).

Thus every subdirectly irreducible algebra in RICRL may be viewed as a union of relative Stone algebras $\boldsymbol{A}(y), y \in Y$, indexed by the positive cone $Y$ of a Sugihara monoid $\boldsymbol{A}_{S u g}$. Some of the relative Stone algebras $\boldsymbol{A}(y)$ may be trivial (with universe $\{y\}$ ). The Sugihara monoid $\boldsymbol{A}_{S u g}$ may also be trivial, in which case $\boldsymbol{A}$ itself is a relative Stone algebra, and in a PSM, each of the relative Stone algebras is a generalized Boolean algebra on two elements, except that $\boldsymbol{A}(e)$ may be trivial.

\section{Applichtions}

Since the variety of positive Sugihara monoids, PSM, is strictly contained in RICRL, it is of interest to distinguish these classes axiomatically. We shall axiomatize PSM equationally, using Theorem [21. We take advantage of the following observation of Meyer and Parks 32 .

Proposition 22 ([32]). Every Sugihara monoid satisfies

$$
[(((x \rightarrow y) \rightarrow y) \rightarrow x) \rightarrow z] \cdot[(((y \rightarrow x) \rightarrow x) \rightarrow y) \rightarrow z] \leq z .
$$

Consequently, every positive Sugihara monoid satisfies this law. We prove that within RICRL, the converse is also true.

Theorem 23. A representable idempotent CRL is a positive Sugihara monoid iff it satisfies (16). 3

\footnotetext{
${ }^{3}$ Meyer and Parks' results and arguments in 32 make Theorem 23 a natural conjecture, but they do not show it to be true. See 11 for a discussion of this point and for axiomatizations of other subreducts of Sugihara monoids.
} 
Proof. By Birkhoff's subdirect decomposition theorem, it suffices to show that any subdirectly irreducible algebra in RICRL that satisfies (16) belongs to PSM. Let $\boldsymbol{A} \in \mathrm{RICRL}_{\mathrm{SI}}$, so $\boldsymbol{A}$ is a linear idempotent CRL, and assume that $\boldsymbol{A}$ satisfies (16). Let $Y=\{a \in A: e \leq a\}$ and for each $y \in Y$, let

$$
A_{y}=\{c \in A: c<e \text { and }|c|=y\} .
$$

Let $y \in Y$. By Theorem 21(iii), it is enough to show that $\left|A_{y}\right| \leq 1$ and that $A_{y} \neq \emptyset$ unless $y=e$.

We show first that $\left|A_{y}\right| \leq 1$. Suppose, on the contrary, that $c, d \in A_{y}$ with $c \neq d$, say $c<d$. Then, in the notation of Theorem 20, we have

$$
c<d \leq y^{\prime}=-y<e \text { and } y=c^{*}=d^{*}=-c=-d=|c|=|d| .
$$

By part (vil) of the same theorem,

$$
\begin{array}{r}
c \rightarrow d=c^{*} \vee d=y \vee d=y, \\
y \rightarrow d=(-y) \wedge d=d, \\
d \rightarrow c=(-d) \wedge c=y \wedge c=c .
\end{array}
$$

So if we define $a=((c \rightarrow d) \rightarrow d) \rightarrow c$ and $b=((d \rightarrow c) \rightarrow c) \rightarrow d$, then

$$
a=(y \rightarrow d) \rightarrow c=d \rightarrow c=c \text { and } b=(c \rightarrow c) \rightarrow d=y \rightarrow d=d .
$$

Now (16) gives $(a \rightarrow d) \cdot(b \rightarrow d) \leq d$. But

$$
(a \rightarrow d) \cdot(b \rightarrow d)=(c \rightarrow d) \cdot(d \rightarrow d)=y \cdot y=y \not \leq d .
$$

This contradiction shows that $\left|A_{y}\right| \leq 1$.

It remains only to show that $A_{y} \neq \emptyset$, unless $y=e$. So let $e<y$ and suppose $A_{y}=\emptyset$. Then

$$
e<y<\uparrow y=(-y)^{*} \quad \text { and } \quad-y=-\uparrow y=(\uparrow y)^{\prime}<e,
$$

since $A_{\uparrow y} \neq \emptyset$. Using Theorem 20 vil) again, we observe that

$$
(-y) \rightarrow e=(-y)^{*} \vee e=(\uparrow y) \vee e=\uparrow y .
$$

Define $a=((e \rightarrow y) \rightarrow y) \rightarrow e$ and $b=((y \rightarrow e) \rightarrow e) \rightarrow y$. Then

$$
a=(y \rightarrow y) \rightarrow e=y \rightarrow e=(-y) \wedge e=-y,
$$

and

$$
b=((-y) \rightarrow e) \rightarrow y=(\uparrow y) \rightarrow y=(-\uparrow y) \wedge y=-y .
$$

By (16), $(a \rightarrow e) \cdot(b \rightarrow e) \leq e$. But

$$
(a \rightarrow e) \cdot(b \rightarrow e)=((-y) \rightarrow e)^{2}=(-y) \rightarrow e=\uparrow y \not \leq e .
$$

From this contradiction we infer that $A_{y} \neq \emptyset$ unless $y=e$, as required.

The identity (16) therefore captures exactly the lingering effect of involution on distributive idempotent CRLs. This identity fails in the algebra $\boldsymbol{E}$ in Example 6 , i.e., $\boldsymbol{E}$ cannot be embedded into an idempotent distributive CRL with an involution. In contrast, every CRL that is square increasing (or square decreasing) and distributive may be embedded into an involutive algebra with just the same properties; see 23] and its references. The next result shows that the lingering effect of involutions on RICRLs is limited to their residuation properties. 
Theorem 24. Let $\boldsymbol{A}=\langle A ; \cdot, \rightarrow, \wedge, \vee, e\rangle$ be an RICRL. Then the lattice-ordered monoid $\langle A ; \cdot, \wedge, \vee, e\rangle$ can be embedded into the corresponding reduct of a Sugihara monoid.

Proof. Recall that every algebra embeds into an ultraproduct of finitely generated subalgebras of itself. In view of this, and Birkhoff's subdirect decomposition theorem, it is enough to prove the present result in the case where $\boldsymbol{A}$ is finitely generated and subdirectly irreducible (hence finite and linear). In discussing $\boldsymbol{A}$, we adopt the notation of Theorem 20 and its proof. We want to replace the operation - by an involution $\neg$, by adding extra elements where necessary. Therefore we extend $\langle A ; \leq\rangle$ to a longer chain $\langle B ; \leq\rangle$ as follows. Whenever $y \in Y$ and $\left|A_{y}\right| \neq \emptyset$, insert a copy $\left\{\neg z: z \in A_{y} \backslash\left\{y^{\prime}\right\}\right\}$ of the dual of $\left\langle A_{y} \backslash\left\{y^{\prime}\right\} ; \leq\right\rangle$ above $y$ and below all upper bounds of $y$; also define $\neg\left(y^{\prime}\right)=y, \neg y=y^{\prime}$ and $\neg \neg z=z$ for all $z \in A_{y}$. Whenever $y \in Y$ and $A_{y}=\emptyset$, add a new element $\neg y$ above $(\uparrow y)^{\prime}$ and below all upper bounds of $(\uparrow y)^{\prime}$; also define $\neg \neg y=y$, so the unary function $\neg$ is defined on all of $B$. We introduce a new 'absolute value function' [ ] on $B$ by defining $[y]=y$ if $e \leq y$ and $[y]=\neg y$, otherwise.

Recall the definition of the total order $\preceq$ on $A$ in the proof of Theorem 20 $((\mathrm{II}) \Rightarrow(\mathrm{I}))$. Similarly, we define a total order $\sqsubseteq$ on $B$ by

$$
\begin{aligned}
& \text { if } u, v \in A \text { and }[u]<[v], \text { then } v \sqsubset u \text {; } \\
& \text { if } u, v \in A \text { and }[u]=[v] \text {, then }(u \sqsubseteq v \text { iff } u \leq v) .
\end{aligned}
$$

It can be verified that the restriction of $\sqsubseteq$ to $A \times A$ is just $\preceq$. So the minimum operation of $\sqsubseteq$ on $B$ is an extension of the operation $\cdot$ of $\boldsymbol{A}$, which we shall also denote by $\cdot$. As in the proof of Theorem 20, $\langle B ; \cdot, e, \leq\rangle$ is a lattice-ordered commutative idempotent monoid which is residuated and involutive, i.e., it is the reduct of a (linear) Sugihara monoid $\boldsymbol{B}$.

Thus, the lattice-ordered monoid reducts of RICRLs and those of involutive RICRLs cannot be distinguished by any universal laws. More precisely:

Corollary 25. RICRL and SM satisfy the same universal first-order sentences in the signature $\{\cdot, \wedge, \vee, e\}$.

Corollary 25 would become false if we by replaced RICRL by the larger variety of distributive idempotent CRLs. To see this, note that the identity

$$
(x \wedge y) \cdot z \approx(x \cdot z) \wedge(y \cdot z)
$$

holds in all linear CRLs, because there $x \wedge y$ takes the value of $x$ or that of $y$, and - preserves the order. Therefore, (17) is satisfied in RICRL. But it is not satisfied in all idempotent distributive CRLs. Indeed, the four-element distributive lattice on $\{0, a, e, 1\}$ generated by incomparable atoms $a$ and $e$ is the lattice reduct of an idempotent CRL with identity $e$, in which $a \cdot 1=1$ and $0 \cdot 1=0$. (All other relations are forced.) In this CRL, we have $(a \wedge e) \cdot 1=0 \cdot 1=0$, while $(a \cdot 1) \wedge(e \cdot 1)=1 \wedge 1=1$.

As we observed in the introduction, we may infer from Corollary 19 that:

Theorem 26. Every finitely based extension of the positive relevant logic $\mathbf{R}_{+}$containing the mingle and Gödel-Dummett axioms has a solvable deducibility problem.

Recall that the mingle and Gödel-Dummett axioms are $\vdash p \rightarrow(p \rightarrow p)$ and $\vdash(p \rightarrow q) \vee(q \rightarrow p)$, respectively. 
The $\mathbf{R}$ of the present paper is the $\mathbf{R}^{t}$ of [2], which was renamed $\mathbf{R}$ in 3 . So $\mathbf{R}$ and its negation-less fragment $\mathbf{R}_{+}$are formulated with the 'Ackermann constant' $t$, which corresponds to $e$. The most important feature of $\mathbf{R}$ is that its theorems exclude the law of weakening $p \rightarrow(q \rightarrow p)$. It is well known that $\mathbf{R}$ and $\mathbf{R}_{+}$have a common deduction theorem, viz., $\Gamma, \varphi \vdash \psi$ iff $\Gamma \vdash(\varphi \wedge t) \rightarrow \psi$. They may therefore be identified for practical purposes with their sets of derivable rules, i.e., with the deducibility relations of the formal systems that generate their sets of theorems. In the case of $\mathbf{R}_{+}$, that formal system is set out for instance on page 40 of [30] and its relation to $\mathbf{R}$ is justified by arguments in [31. The traditional 'logics over $\mathbf{R}_{+}$' are the sets of theorems generated by axiomatic extensions of this formal system.

In Theorem 26. however, extensions need not be assumed axiomatic and the strongest reading of the result is obtained by identifying them with the consequence relations in the signature $\cdot, \rightarrow, \wedge, \vee, t$ that contain all derivable rules of $\mathbf{R}_{+}$. We understand by a 'consequence relation' any finitary and structural deductive system in the sense, for instance, of [9].

To be more precise about 'fragments': a fragment of a consequence relation $\vdash$ is just the set of all derivable rules of $\vdash$ that are formulated in a specified subset of the signature. It is a consequence relation again, so the notion of axiomatization is already defined for it; i.e., an axiomatization is any formal system whose deducibility relation is the consequence relation under discussion.

Dunn's results in 2 show that the class of de Morgan monoids, which is a variety, is the equivalent algebraic semantics for $\mathbf{R}$, in the sense of 9 . A formula $\varphi$ is a theorem of $\mathbf{R}$ iff the identity $e \leq \varphi$ holds in all de Morgan monoids, where of course the latter abbreviates $e \approx e \wedge \varphi$. Conversely, an equation $\alpha \approx \beta$ is an identity of de Morgan monoids iff the formula $\alpha \leftrightarrow \beta$ is a theorem of $\mathbf{R}$, where $p \leftrightarrow q$ abbreviates $(p \rightarrow q) \wedge(q \rightarrow p)$. These translations extend naturally to correspondences between the derivable rules $\Gamma \vdash \varphi$ of $\mathbf{R}$ and the valid quasi-identities of de Morgan monoids. The translations are mutually inverse, because $p \dashv \vdash_{\mathbf{R}} t \leftrightarrow(t \wedge p)$, and de Morgan monoids satisfy $\alpha \approx \beta \Longleftrightarrow e \leq \alpha \leftrightarrow \beta$.

In this situation, 9, Cor. 4.9, Thm. 2.17] shows that the logical extensions of the algebraizable system must match the subquasivarieties of the equivalent algebraic semantics in the same way, and axiomatic extensions must match subvarieties. Also, finite axiomatizability is preserved and reflected. Finally, a fragment of an algebraizable consequence relation $\vdash$ is itself algebraized by the matching subreduct class of the equivalent algebraic semantics for $\vdash$, provided that the connectives used in the translations survive. This is [9, Cor. 2.12].

Since every square increasing distributive CRL may be embedded into a de Morgan monoid (see [31] or [23]), the variety of square increasing distributive CRLs is the equivalent algebraic semantics for $\mathbf{R}_{+}$, and the extension of $\mathbf{R}_{+}$by mingle and the Gödel-Dummett axiom is algebraized in the same way by RICRL. Further extensions correspond similarly to the subquasivarieties of RICRL, and Theorem 26 therefore follows from Corollary 19.

In all of these correspondences, the failure of the weakening law manifests itself in the fact that the algebras need not be integral, even when the mingle and GödelDummett axioms are added.

On the same grounds, Theorem 23 has the following logical interpretation, which was previously a plausible hypothesis. Here $\varphi \rightarrow \psi \rightarrow \eta$ abbreviates $\varphi \rightarrow(\psi \rightarrow \eta)$. 
Proposition 27. The negation-less fragment of $\mathbf{R M}^{t}$ is axiomatized, relative to $\mathbf{R}_{+}$, by the mingle and Gödel-Dummett axioms together with the Meyer-Parks axiom

$$
\vdash[(((p \rightarrow q) \rightarrow q) \rightarrow p) \rightarrow r] \rightarrow[(((q \rightarrow p) \rightarrow p) \rightarrow q) \rightarrow r] \rightarrow r .
$$

The final result confirms that the hereditary character of Theorem[26 has genuine applications. It shows that Theorem [26] applies to infinitely many axiomatic and finitely based extensions of $\mathbf{R}_{+}$that are strictly weaker than the negation-less fragments of both $\mathbf{R M}^{t}$ and the Gödel-Dummett logic $\mathbf{L C}$ of [16].

Theorem 28. There are infinitely many finitely axiomatized varieties of RICRLs strictly containing the variety generated by all relative Stone algebras together with all positive Sugihara monoids.

Proof. Let $\mathrm{V}$ denote the variety generated by RSA $\cup$ PSM.

We construct a sequence of subdirectly irreducible idempotent linear CRLs $\boldsymbol{A}^{3}$, $\boldsymbol{A}^{4}, \ldots$, using the notational conventions of Theorem 21] For each $n>2$, the positive cone $Y^{n}$ of the algebra $\boldsymbol{A}^{n}$ is a chain $e=0<1<\cdots<n$, and $A_{n}^{n}$ is a singleton, so $\boldsymbol{A}^{n}(n)$ is a two-element algebra. But for $m<n$, the set $A_{m}^{n}$ is empty; hence $\boldsymbol{A}^{n}(m)$ is trivial and $\boldsymbol{A}_{\text {Sug }}^{n} \cong \boldsymbol{Z}_{3}^{\urcorner}$. In fact $\boldsymbol{A}^{3}$ is just the algebra $\boldsymbol{E}$ of Example 6. We note that each $\boldsymbol{A}^{n}$ is a simple algebra. By the congruence extension property for CRLs, the nontrivial subalgebras of $\boldsymbol{A}^{n}$ are simple also.

For each $n$, let $\mathrm{K}_{n}$ be the class consisting of all the linear members of RSA $\cup$ PSM together with all algebras that can be embedded into $\boldsymbol{A}^{n}$, i.e., we adjoin the class $\operatorname{IS}\left(\boldsymbol{A}^{n}\right)$. Note that $\mathrm{S}\left(\boldsymbol{A}^{n}\right)$ is a finite set of finite algebras of finite type, so embeddability into $\boldsymbol{A}^{n}$ can be expressed by a first-order sentence. Since linearity is also a single first-order sentence

$$
\forall x \forall y(x \approx x \wedge y \text { or } y \approx y \wedge x)
$$

and since RSA and PSM are finitely axiomatized varieties, membership of $\mathrm{K}_{n}$ is definable by a single first-order sentence as well.

It follows that $\mathrm{K}_{n}$ is closed under ultraproducts, and it is obviously closed under subalgebras, as (18) is a universal sentence. Since (18) is also a positive sentence, it persists under homomorphisms as well. Embeddability into $\boldsymbol{A}^{n}$ is trivially preserved by homomorphisms, because every nontrivial subalgebra of $\boldsymbol{A}^{n}$ is simple. So, since RSA and PSM are varieties, it follows that $\mathrm{K}_{n}$ is closed under homomorphic images.

We have shown that $\mathrm{K}_{n}$ is closed under the class operators $\mathrm{H}, \mathrm{S}$ and $\mathrm{P}_{\mathrm{U}}$, so it is axiomatizable by a set of positive universal sentences. Since it is also axiomatized by a single first-order sentence, the Compactness Theorem of first-order logic implies that $\mathrm{K}_{n}$ can be axiomatized by finitely many positive universal sentences. Then, since RICRL is finitely axiomatized and has EDPC, it follows from Theorem 4 that the variety $\mathrm{V}\left(\mathrm{K}_{n}\right)$ is also finitely axiomatized (by equations).

It is easy to see that when $m<n$, then $\boldsymbol{A}^{m}$ embeds in $\boldsymbol{A}^{n}$, so the varieties $\mathrm{V}\left(\mathrm{K}_{n}\right)$ form a chain. In Example 6 we showed that $\boldsymbol{A}^{3}$ does not belong to $\mathrm{V}$. Suppose $2<$ $m<n$. Since $\boldsymbol{A}^{m}$ is simple, Jónsson's Lemma shows that the subdirectly irreducible members of $\mathrm{V}\left(\mathrm{K}_{m}\right)$ are just the nontrivial subalgebras of $\boldsymbol{A}^{m}$ together with certain algebras in RSA $\cup$ PSM, and this class excludes the subdirectly irreducible algebra $\boldsymbol{A}^{n}$. Thus the varieties $\mathrm{V}\left(\mathrm{K}_{n}\right)$ are distinct for different values of $n$; i.e., they form an infinite chain of supervarieties of $\mathrm{V}$ within RICRL. 
Remark. In the proof that $\mathrm{V}\left(\mathrm{K}_{n}\right)$ is finitely axiomatized, the use of EDPC could be eliminated. Baker's Finite Basis Theorem [14, Cor. V.4.18] says that every finitely generated congruence distributive variety of finite type is finitely axiomatized; this applies to $\mathrm{V}\left(\boldsymbol{A}^{n}\right)$. Since PSM and RSA are also finitely based, we can apply a result of Galatos [20, which says that the join of finitely many finitely axiomatized varieties of CRLs is still finitely axiomatized. (An arbitrary variety of CRLs need not have EDPC.)

\section{REFERENCES}

[1] P. Aglianò, Ternary deductive terms in residuated structures, Acta Sci. Math. (Szeged) 64 (1998), 397-429. MR.1666002 (2000a:08005)

[2] A.R. Anderson, N.D. Belnap, Jr., "Entailment: The Logic of Relevance and Necessity, Volume 1", Princeton University Press, 1975. MR0406756 (53:10542)

[3] A.R. Anderson, N.D. Belnap, Jr., J.M. Dunn, "Entailment: The Logic of Relevance and Necessity, Volume 2", Princeton University Press, 1992. MR,1223997 (94b:03042)

[4] A. Avron, The semantics and proof theory of linear logic, Theoretical Computer Science $\mathbf{5 7}$ (1988), 161-184. MR0960102 (90k:68100)

[5] R. Balbes, P. Dwinger, "Distributive Lattices", University of Missouri Press, Columbia, 1974. MR0373985 (51:10185)

[6] W.J. Blok, W. Dziobiak, On the lattice of quasivarieties of Sugihara algebras, Studia Logica 45 (1986), 275-280. MR0877316 (88d:08005)

[7] W.J. Blok, I.M.A. Ferreirim, On the structure of hoops, Algebra Universalis 43 (2000), 233257. MR.1774741(2001g:03120)

[8] W.J. Blok, D. Pigozzi, On the structure of varieties with equationally definable principal congruences I, Algebra Universalis 15 (1982), 195-227. MR0686803 (85g:08005)

[9] W.J. Blok, D. Pigozzi, "Algebraizable Logics", Memoirs of the American Mathematical Society, Number 396, Amer. Math. Soc., Providence, 1989. MR0973361 (90d:03140)

[10] W.J. Blok, J.G. Raftery, Constructing simple residuated lattices, Algebra Universalis 50 (2003), 385-389. MR2055065 (2005a:06017)

[11] W.J. Blok, J.G. Raftery, Fragments of R-mingle, Studia Logica 78 (2004), 59-106. MR2108021 (2005i:03027)

[12] W.J. Blok, C.J. van Alten, The finite embeddability property for residuated lattices, pocrims and BCK-algebras, Algebra Universalis 48 (2002), 253-271. MR.1954775 (2003j:06017)

[13] W.J. Blok, C.J. van Alten, On the finite embeddability property for residuated ordered groupoids, Trans. Amer. Math. Soc. 357 (2005), 4141-4157. MR2159703

[14] S. Burris, H.P. Sankappanavar, "A Course in Universal Algebra", Graduate Texts in Mathematics, Springer-Verlag, New York, 1981. MR0648287(83k:08001)

[15] C.C. Chang, Algebraic analysis of many valued logic, Trans. Amer. Math. Soc. 88 (1958), 467-490. MR0094302 (20:821)

[16] M. Dummett, A propositional calculus with a denumerable matrix, J. Symbolic Logic 24 (1959), 97-106. MR0123476 (23:A801)

[17] J.M. Dunn, Algebraic completeness results for $R-$ mingle and its extensions, J. Symbolic Logic 35 (1970), 1-13. MR0288008 (44:5206)

[18] K. Dyrda, None of the variety $E_{n}, n \geq 2$, is locally finite, Demonstratio Math. 20 (1987), 215-219. MR0941416 (89b:06007)

[19] N. Galatos, "Varieties of Residuated Lattices", Ph.D. Thesis, Vanderbilt University, 2003

[20] N. Galatos, Equational bases for joins of residuated-lattice varieties, Studia Logica 76 (2004), 227-240. MR2072984 (2005f:06019)

[21] N. Galatos, Minimal varieties of residuated lattices, Algebra Universalis 52 (2005), 215-239. MR2161651 (2006f:06010)

[22] N. Galatos, H. Ono, Algebraization, parametrized local deduction theorem and interpolation for substructural logics over FL, Studia Logica, 83 (2006), 279-308. MR.2250112

[23] N. Galatos, J.G. Raftery, Adding involution to residuated structures, Studia Logica 77 (2004), 181-207. MR2080238 (2005f:06020)

[24] J. Hart, L. Rafter, C. Tsinakis, The structure of commutative residuated lattices, Internat. J. Algebra Comput. 12 (2002), 509-524. MR1919685 (2003g:06008) 
[25] P. Jipsen, C. Tsinakis, A survey of residuated lattices, in "Ordered Algebraic Structures", edited by J. Martinez, Kluwer, Dordrecht, 2002, pp. 19-56. MR2083033 (2005e:06024)

[26] B. Jónsson, Algebras whose congruence lattices are distributive, Math. Scand. 21 (1967), 110-121. MR0237402(38:5689)

[27] J. Łoś, R. Suszko, Remarks on sentential logics, Proc. Kon. Nederl. Akad. van Wetenschappen, Series A 61 (1958), 177-183. MR0098670(20:5125)

[28] A.I. Mal'cev, "Algebraic Systems", Springer-Verlag, Berlin, 1973. MR0349384 (50:1878)

[29] R.K. Meyer, A characteristic matrix for RM, unpublished manuscript, 1967. Partly subsumed in [2, Sec. 29.3].

[30] R.K. Meyer, Conservative extension in relevant implication, Studia Logica 31 (1972), 39-46. MR0381937 (52:2826)

[31] R.K. Meyer, On conserving positive logics, Notre Dame J. Formal Logic 14 (1973), 224-236. MR0327471 (48:5813)

[32] R.K. Meyer, R.Z. Parks, Independent axioms for the implicational fragment of Sobocinsski's three-valued logic, Zeitschr. f. math. Logik und Grundlagen der Math. 18 (1972), 291-295. MR0357062 (50:9530)

[33] I. Nishimura, On formulas of one variable in intuitionistic propositional calculus, J. Symbolic Logic 25 (1960), 327-331. MR0142456 (26:25)

[34] J.S. Olson, Subdirectly irreducible residuated semilattices and positive universal classes, Studia Logica, 83 (2006), 393-406. MR2250117

[35] J.S. Olson, J.G. Raftery, Positive Sugihara monoids, Algebra Universalis, to appear.

[36] H. Ono, Proof-theoretic methods in nonclassical logic - an introduction, in: M. Takahashi, M. Okada and M. Dezani-Ciancaglini (eds.), "Theories of Types and Proofs", MSJ Memoirs 2, Mathematical Society of Japan, 1998, pp. 207-254. MR.1728763 (2000k:03125)

[37] H. Ono, Substructural logics and residuated lattices - an introduction, in V.F. Hendricks, J. Malinowski (eds.), "50 Years of Studia Logica", Trends in Logic, vol. 20, Kluwer, 2003, pp. 177-212. MR2045284 (2004k:03046)

[38] L. Rieger, Zametka o t. naz. svobodnyh algebrah s zamykanijami, Czechoslovak Math. J. 7 (82) (1957), 16-20. MR0091266 (19:938c)

[39] P. Schroeder-Heister, K. Došen (eds.), "Substructural Logics", Clarendon Press, Oxford, 1993. MR1283190 (95a:03003)

[40] T. Sugihara, Strict implication free from implicational paradoxes, Memoirs of the Faculty of Liberal Arts, Fukui University, Series I (1955), 55-59.

[41] A.S. Troelstra, "Lectures on Linear Logic", CSLI Lecture Notes No. 29, 1992. MR.1163373 (93i:03083)

[42] C.J. van Alten, The finite model property for knotted extensions of propositional linear logic, J. Symbolic Logic 70 (2005), 84-98. MR2119124 (2005m:03127)

[43] C.J. van Alten, J.G. Raftery, Rule separation and embedding theorems for logics without weakening, Studia Logica 76 (2004), 241-274. MR2072985 (2005e:03132)

School of Mathematical Sciences, University of KwaZulu-Natal, Durban 4001, South AFrica

E-mail address: raftery@ukzn.ac.za 